\title{
La función discursiva y la distribución social de los marcadores por ser y onda en el corpus del PRESEEA de Santiago de Chile
}

\author{
Abelardo San Martín Núñez ${ }^{1}$ \\ Cristian Rojas Inostroza \\ Silvana Guerrero González \\ Universidad de Chile
}

\begin{abstract}
RESUMEN
Los estudios sobre los denominados "marcadores del discurso" han coincidido en destacar su importancia para la producción y la comprensión del discurso, tanto oral como escrito, en diferentes lenguas. Pese a su importancia, una de las dimensiones menos indagadas en la lengua española es la variación social y dialectal del uso de las mencionadas partículas. De este modo, la mayor parte de las aproximaciones a las funciones discursivas de los marcadores ha tendido a basarse en muestras del español peninsular y en ejemplos reconstruidos o de la lengua escrita. En este estudio, se analiza el funcionamiento pragmático y la estratificación sociolingüística de dos marcadores empleados en el español hablado en Santiago de Chile: por ser y onda. Los objetivos de este artículo son identificar las funciones que cumplen dichos marcadores del discurso y correlacionar sus frecuencias de empleo con las variables sociodemográficas sexo,
\end{abstract}

\footnotetext{
Para correspondencia, dirigirse a: Abelardo San Martín (asmartin@uchile.cl), Cristian Rojas (rojascristian@ug.uchile.cl) o a Silvana Guerrero (siguerrero@u.uchile.cl), Departamento de Lingüística, Facultad de Filosofía y Humanidades, Universidad de Chile, Av. Capitán Ignacio Carrera Pinto 1025, Ñuñoa, Santiago.
} 
edad y nivel de estudios de los informantes. Para tal propósito, se analizará el comportamiento de estas partículas en las entrevistas que conforman el corpus sociolingüístico del PRESEEA de Santiago.

Palabras clave: marcadores del discurso, variación discursiva, sociolingüística, español de Chile.

THE DISCOURSE FUNCTION AND THE SOCIAL DISTRIBUTION OF THE MARKERS POR SER AND ONDA IN THE PRESEEA CORPUS From Santiago de Chile

\begin{abstract}
Studies about "discourse markers" have coincided in stressing their importance to the production and comprehension of discourse, both oral and written, in different languages. Despite their importance, one of the less investigated dimensions in the Spanish language is the social and dialectal variation in the use of the aforementioned particles. Thus, the majority of the approaches to the discursive functions of the markers have a tendency to be based on peninsular Spanish and on reconstructed examples or on written language. In this study, the pragmatic functioning and sociolinguistic stratification of two markers used in the Spanish spoken in Santiago, Chile are analyzed: por ser and onda. The objectives of this article are to identify the functions said discourse markers fulfill and to correlate their use frequency with the sex, age, and level of education sociodemographic variables. For this purpose, the behavior of these particles in the interviews that make up the sociolinguistic corpus of the PRESEEA of Santiago will be analyzed.
\end{abstract}

Keywords: discourse markers, discursive variation, sociolinguistics, Chilean Spanish.

Recibido: $12 / 05 / 2016 \quad$ Aceptado: 06/07/2016

\title{
1. INTRODUCCIÓN
}

Por ser y onda adquieren, en el español de Chile, un valor pragmático más allá de su función sintáctica oracional, permitiendo a los hablantes orientar la comprensión de sus enunciados, de modo que se los puede 
analizar como "marcadores del discurso". Al no ser descritos ni incluidos en las clasificaciones (Martín Zorraquino y Portolés 1999; Portolés 2001; Cortés y Camacho 2005), ni en los diccionarios de partículas más conocidos (DPDE; Fuentes 2009), se los puede caracterizar, desde el punto de vista dialectal, como usos diferenciales del español de Chile, a nivel de discurso, en su variante más coloquial (DUECH, s v. ser y onda). El objetivo general de este estudio consiste en analizar el comportamiento pragmático y la estratificación sociolingüística del empleo de los marcadores del discurso por ser y onda, en las 108 entrevistas que conforman el corpus de Santiago de Chile del Proyecto para el Estudio Sociolingüístico del Español de España y América (PRESEEA-SA) ${ }^{2}$. De manera específica, pretendemos: a) identificar las funciones que cumplen dichos marcadores del discurso en la muestra seleccionada y b) correlacionar sus frecuencias de empleo con los factores socio-demográficos (sexo, edad y nivel de educacional) de los informantes. Las hipótesis que someteremos a contrastación empírica, en este trabajo, son que los marcadores analizados son polifuncionales y que los factores sociodemográficos de los sujetos inciden con diferente intensidad en su empleo.

\section{MARCO TEÓRICO}

\subsection{LOS MARCADORES DEL DISCURSO EN ESPAÑOL Y SU ESTUDIO SOCIOLINGÜÍSTICO}

Los "marcadores del discurso" son unidades de procesamiento que cumplen una función extra-oracional a nivel semántico-pragmático, controlando la situación de enunciación, organizando la información discursiva y guiando la interpretación de los enunciados por parte de los interlocutores (cf. Martín Zorraquino y Portolés 1999; Portolés 2001)33. El interés por el estudio de

2 El equipo de PRESEEA-SA está compuesto por los profesores de la Universidad de Chile, Alfredo Matus Olivier (coordinador), Abelardo San Martín Núñez y Silvana Guerrero González (investigadores) y Cristian Rojas Inostroza (colaborador).

3 Martín Zorraquino (1998: 35-51) plantea que, pese a la heterogeneidad de los marcadores del discurso, relativa tanto a las categorías a las que pertenecen como a su distribución en el discurso y a sus funciones pragmáticas, dichas unidades presentan, entre otras, las siguientes propiedades comunes: a) no pueden ser sustituidas por elementos 
estas unidades se ha incrementado de manera notable, en los últimos años, dando origen a un número significativo de obras colectivas que dan cuenta de la importancia de estas partículas en la producción y la comprensión del discurso tanto oral como escrito (cf. Martín Zorraquino y Montolío 1998; Loureda y Acín 2010; Aschenberg y Loureda 2011). En este estudio, adoptaremos, en lo principal, la perspectiva teórica sobre los marcadores del discurso desarrollada en Martín Zorraquino y Portolés (1999), Portolés (2001), Cortés y Camacho (2005) y Fuentes (2009).

Uno de los aspectos menos desarrollados en el marco de la investigación de los marcadores del discurso en lengua española es la dimensión dialectal y social de su empleo (Carbonero y Santana 2010). De este modo, la mayor parte de las aproximaciones a las funciones discursivas de los marcadores ha tendido a basarse en muestras del español peninsular y en ejemplos reconstruidos o de la lengua escrita. Cortés (1998: 160) concluye que el estudio variacionista de los marcadores es posible, si se cumple el requisito de contrastar las frecuencias de conectores que cumplan las mismas funciones, lo que trae aparejadas no pocas dificultades. Para el análisis sociolingüístico de los marcadores, entonces, es preciso que se asocien las características de los informantes con aquellas partículas que desempeñen una función común en el discurso (Cortés 1998: 154). Por su parte, Carbonero y Santana (2010: 516-517) destacan la carencia de trabajos que aborden la variación espacial y social de los marcadores del discurso, aunque -al mismo tiempo-destacan el interés de esta línea de trabajo para el mejor conocimiento de estas unidades de procesamiento.

\subsection{LOS ESTUDIOS SOBRE LOS MARCADORES DEL Discurso EN EL ESPAÑOL DE CHILE}

Por lo que se refiere al estudio de los marcadores del discurso, en general, en el español hablado en Chile, Rabanales y Contreras (1995) estudian las funciones de las "muletillas" en el habla culta de Santiago. Además de proporcionar una clasificación de las funciones de estas unidades en una muestra de diálogos informales de hablantes cultos, los autores entregan datos cuantitativos sobre su empleo, resultando ser los jóvenes y los hombres

pronominales o deícticos, b) no pueden ser sometidos a interrogación parcial, c) no admiten la coordinación con elementos equifuncionales y d) no pueden ser reemplazados por elementos pronominales que reproduzcan la totalidad, o la parcialidad, de la oración en la que se sitúan. 
los que más las emplean. Por otro lado, Pons y Samaniego (1998) estudian los marcadores de apoyo empleados en una muestra de discurso oral de hablantes cultos de Santiago, según tres grupos etarios y la variable sexo (mujer/hombre) en el discurso de 10 sujetos. Más allá de la descripción del habla capitalina, Poblete (1998 y 1999) y Cepeda (1999) estudian los marcadores del discurso empleados en Valdivia, así como Soto y Roldán (2002) los usados en Ñuble.

De modo más reciente, en San Martín (2004-2005) estudiamos la función pragmática y la estratificación sociolingüística del empleo de la partícula igual como reformulador de distanciamiento (Portolés 2001), en comparación con otros marcadores que cumplían la misma función, a saber, de todas maneras y en todo caso, confirmando la preeminencia estadística de igual. La misma estrategia investigativa fue aplicada en San Martín (2011) para el estudio de los marcadores de control de contacto y en San Martín (2013) para los reformuladores de distanciamiento en el corpus PRESEEA-SA. Asimismo, en Rojas et al. (2012) estudiamos los marcadores de reformulación empleados en una muestra de 72 hablantes santiaguinos. Los resultados muestran un empleo preponderante de $o$ sea, tanto con valor explicativo como rectificativo, de igual como reformulador de distanciamiento y de al final con función recapitulativa. Además, los factores edad y grupo socioeconómico de los hablantes son más determinantes en el empleo variable de los marcadores relevados.

Por último, Valencia (2014b) informa los resultados de una investigación sobre los marcadores del discurso recogidos en una muestra de 12 entrevistas de hablantes santiaguinos, en el marco del Proyecto de Estudio de la Norma Culta Hispánica y como parte de un estudio coordinado sobre estas partículas, en diferentes urbes de habla española. Además de la limitación de restringirse solo al nivel culto del espectro sociolingüístico de Santiago, el objetivo del proyecto era permitir la comparación de los marcadores, en las diferentes muestras recopiladas; por lo tanto, como señala Valencia (2014a: 7-8), no se relevaron usos idiosincrásicos o dialectales, ya que el análisis se limitó a aplicar la clasificación de marcadores propuesta en Martín Zorraquino y Portolés (1999) ${ }^{4}$.

\footnotetext{
4 Otros marcadores característicos del español de Chile que han sido abordados de modo monográfico son de repente (Rojas 2008), huevón (Rojas 2012) y cachái (Mondaca et al. 2015).
} 


\section{METODOLOGÍA}

\subsection{CORPus}

El corpus que sirvió de base para el análisis del presente estudio consistió en las 108 entrevistas sociolingüísticas que conforman el corpus PRESEEASA. Para la recopilación de dichos materiales, se aprovechó la instancia de aplicación de un instrumento que se realiza anualmente desde 1978 hasta la fecha, en el marco de la asignatura de Sociolingüística que se imparte en las Licenciaturas en Lengua y Literatura Hispánica y Lengua y Literatura Inglesas de la Universidad de Chile. Las entrevistas fueron hechas a hombres y mujeres con características sociodemográficas congruentes, aplicando los supuestos metodológicos sugeridos por Labov (1983 [1972]). Consecuentemente, en dichas situaciones los entrevistadores debían tratar de superar la "paradoja del observador" consiguiendo, de esta forma, una muestra significativa de discurso natural grabado (vernáculo) de hablantes de la comunidad de habla en estudio. En las instrucciones a los entrevistadores, se enfatizó que durante la entrevista debía generarse un clima de confianza, a fin de obtener el estilo de habla más espontáneo o natural del sujeto. Asimismo, se les indicó que, idealmente, los hablantes debían olvidarse de que estaban siendo grabados y debían comportarse de modo espontáneo, como si estuvieran participando en una conversación natural.

\subsubsection{Población de la muestra}

Para la conformación de la muestra, se consideró la población santiaguina que hubiera residido en Santiago la mayor parte de sus vidas, independientemente de que hubiera nacido en dicha ciudad o no. El cuestionario de la entrevista se aplicó a una muestra de sujetos del tipo denominado "muestra por cuotas con afijación uniforme" (López Morales 1994). La muestra así conformada comprende un total de 108 individuos, distribuidos de manera proporcional como se indica en la Tabla 1, que se incluye a continuación. 
Tabla 1: Distribución de sujetos de la muestra por nivel educacional, sexo y edad, según PRESEEA-SA

\begin{tabular}{|l|c|c|c|c|c|c|c|}
\hline \multicolumn{7}{|c|}{ Grupo de edad } \\
\hline $\begin{array}{l}\text { Nivel } \\
\text { educacional }\end{array}$ & \multicolumn{2}{|c|}{$20-34$} & \multicolumn{2}{|c|}{$35-54$} & \multicolumn{2}{|c|}{55 y más } & \multirow{2}{*}{ Totales } \\
\cline { 2 - 8 } & $\mathrm{H}$ & $\mathrm{M}$ & $\mathrm{H}$ & $\mathrm{M}$ & $\mathrm{H}$ & $\mathrm{M}$ & \\
\hline Bajo & 6 & 6 & 6 & 6 & 6 & 6 & 36 \\
\hline Medio & 6 & 6 & 6 & 6 & 6 & 6 & 36 \\
\hline Alto & 6 & 6 & 6 & 6 & 6 & 6 & 36 \\
\hline Totales & 18 & 18 & 18 & 18 & 18 & 18 & 108 \\
\hline
\end{tabular}

\subsubsection{Procedimiento de estratificación empleado}

Siguiendo el sistema de estratificación del Proyecto PRESEEA, se estratificó la muestra de sujetos de acuerdo con sus respectivos niveles de instrucción, es decir, según la equivalencia entre los niveles de estudios básico, secundario y superior con los grupos bajo, medio y alto, respectivamente. Sin embargo, con el propósito de garantizar la homogeneidad en la composición de los distintos grupos de la muestra y, por lo tanto, la representatividad de los mismos en relación con la población analizada, se complementó dicho sistema de estratificación con el procedimiento de estratificación empleado por el proyecto de Estudio Sociolingüístico del Español de Chile (ESECH). Dicho sistema de estratificación considera una escala de estatus socioeconómico que contempla las siguientes variables independientes: nivel educacional, categoría ocupacional y comuna de residencia (para más detalles, cf. San Martín y Guerrero 2015).

\section{2. Procedimiento analítico}

Nuestro análisis de la función discursiva y la distribución social de los marcadores por ser y onda se concentró en la determinación de su comportamiento pragmático y de sus frecuencias de ocurrencia, en la muestra recopilada, la que fue revisada en su totalidad. Asimismo, de modo coherente con nuestros objetivos, se observó la incidencia de los siguientes factores sociodemográficos de los hablantes: sexo, edad y nivel educacional. Por lo que se refiere a la descripción de los procedimientos estadísticos aplicados, en este trabajo, seguimos las sugerencias e indicaciones de Moreno Fernández (1990), López Morales (1994) y Hernández Campoy y Almeida (2005). 
Nuestro análisis estadístico atenderá a dos niveles de observación: a) en términos descriptivos, según las frecuencias absolutas y los porcentajes de frecuencia de cada marcador y b) en términos interpretativos o inferenciales, con base en la comparación entre las medias o tendencias centrales de los datos 5 .

\section{ANÁLISIS DE RESULTADOS}

\subsection{ANÁLISIS DISCURSIVO DE LOS MARCADORES POR SER Y ONDA EN EL CORPUS PRESEEA-SA}

La locución por ser y el lexema onda se emplean, en algunas ocasiones, en el español de Chile, más allá del ámbito predicativo oracional, con el objetivo de orientar las inferencias de los interlocutores, esto es, como partículas discursivas o marcadores del discurso. Este "significado de procesamiento" de por ser y onda lo podemos verificar en los ejemplos 1 a 4, a continuación:

\section{Por ser}

1) I.: ay que es pésimo // que es horrendo porque hay gente que viaja lejos / lejos / por ser si voy a determinado lugar tengo que tomar tres locomociones y subir las escalas horribles en los metros (SCHI_M32_070)

5 El paquete estadístico al que hemos recurrido para la estadística inferencial es el SPSS (Statistical Package for the Social Sciences), versión 15.0 para Windows, específicamente, la prueba Análisis de varianza ANOVA. El grado de significación se definirá en el 5\%, según el cual $\mathrm{p}=<0,05$ será estadísticamente significativo.

${ }^{6}$ El código de los sujetos tiene las siguientes equivalencias: SCHI: Santiago de Chile; $\mathrm{Sexo}=\mathrm{H}$ (hombre) y M (mujer). El primer número que le sigue a dichas equivalencias corresponde al código del grupo de edad: $1=20$ a 34 años, $2=35$ a 54 años y $3=55$ años y más; el segundo número corresponde al nivel educacional: $1=$ Bajo (sin estudios o con instrucción primaria o instrucción secundaria incompleta), $2=$ Medio (con instrucción secundaria) y $3=$ Alto (con instrucción superior). Los dígitos finales corresponden a numeración correlativa que se le asignó a los sujetos en el corpus PRESEEA Santiago de Chile. Respecto de la transcripción de los ejemplos, es necesario señalar que: 1) se emplearon las siguientes convenciones para señalar a los participantes de las entrevistas del corpus: $\mathrm{E}=$ entrevistador e I = informante; 2) el texto correspondiente a cada ejemplo de las entrevistas se transcribe en ortografía convencional, incluidos los acentos gráficos; 3) para la mejor comprensión de los ejemplos que hemos incluido en este artículo, se ha suprimido el sistema de etiquetas pertenecientes al Standard Generalized Markup Language (SGML) contenidas en el Text Encoding Initiative 
2) I.: eran bonitas / en los barrios por ser en el barrio que yo viví / se cerraban los pasajes / las esquinas y se engalanaban con guirnaldas / con todo eso / la gente sacaba sus radios / sus equipos que eran tocadiscos de la época (SCHI_H32_065);

\section{Onda}

3) y por otro lado también pasaba lo mismo con no sé po con las drogas por ejemplo obviamente ¿cacháis? onda los huevones que fumaban que fumaban yerba eran los locos más como // como flaites (SCHI_H13_074);

4) viviría onda / no sé / en PF alguna huevada así (SCHI_H13_074).

Los ejemplos anteriores contrastan con el empleo de "por ser" y "onda" dentro del ámbito predicativo oracional, esto es, ya no como marcadores del discurso, sino que como formantes sintagmáticos cuyo contenido léxico contribuye al significado de la oración en la que se incorporan, como se muestra en los ejemplos 5 a 9:

5) I.: pero resulta que mira no es por ser discriminatorio ni nada pero ha llegado mucho peruano a ese barrio / y mucha gente por lo mismo se ha ido / hay mucha gente peruana que es muy buena muy amable pero hay mucha que son muy <silencio/> peleadores (SCHI_H11_006);

6) I.: es lo más / lo más lindo que le pasa a uno como gente / por ser pobre es una casa po / tener su casita propia (SCHI_M21_022);

7) I.: no no llamé nada porque no sé / me dio lata me dio por la mujer esta me dio cosa por la $\mathrm{M}$ / la $\mathrm{M}$ está acostumbrada sabe lo que hace su hijo pero el cabro no sé / como andan en la onda de robar para las drogas todo / ahora estamos más tranquilos porque está preso (SCHI_M31_036);

8) I.: sí / porque ella es súper eeh mi mamá su regalona es mi tía $\mathrm{M}$ es así como la más pero mi tía $\mathrm{P}$ es como ella es es si si no tiene nada y tiene un kilo de pan y tiene pan con mantequilla ella feliz tú te vayas vayas a ver ella lo que haiga nomás ella te atiende igual / o sea da lo mismo ella atiende súper bien / siempre te da consejos y te atiende siempre con una sonrisa y es como súper buena onda (SCHI_M11_009);

9) I.: y nada po / y me di vuelta y le dije así como le dije "oye ¿qué onda?" y la huevada y el huevón se puso así como súper bueno a mí me dio más

(TEI), que se emplean en la transcripción de las entrevistas pertenecientes al PRESEEA, excepto las pausas y los silencios. 
vergüenza que a él po huevón // pero no es / bueno eso puede ser como una anécdota ¿o no? (SCHI_M11_010).

En los ejemplos anteriores, "por ser" es una frase preposicional que asume un valor circunstancial, mientras que "onda" es un lexema con el significado de "tendencia", "manera" o "estilo" o un formante de las unidades fraseológicas: buena onda y ¿qué onda? Al respecto, el DA registra onda "manera, gusto, estilo de una persona" como americanismo que se emplea en Chile y en otros países; esa onda para manifestar sorpresa ante algo que se ha dicho y ¿qué onda? para preguntar qué ocurre y como fórmula de saludo (s. v. onda). El DUECH también incluye la locución adjetiva buena onda "de carácter simpático" y la fórmula ¿qué onda? usada por un hablante "para instar al interlocutor a informarle de algo" (s. v. onda).

El origen del empleo de por ser y onda como marcadores del discurso podemos atribuirlo a procesos de gramaticalización o discursivización, que se explican con relativa facilidad. Por una parte, en por ser es determinante la incidencia del verbo copulativo ser, puesto que también lo ha sido para otros marcadores, con los que comparte ese rasgo en común, como o sea, es decir y esto es, que además, como veremos más adelante, cumplen funciones semejantes. Por otro lado, en onda podemos suponer la discursivización de un sustantivo, la que también ha operado para la generación de otros marcadores como hombre o mujer, en español peninsular, o como huevón, en el español de Chile.

Aunque no le atribuye un significado pragmático, el DA señala que por ser es una locución conjuntiva que, en Chile, equivale a "esto es, a saber" (s. v. ser), es decir, reconoce de modo implícito su valor reformulativo explicativo. El DUECH, en cambio, recoge ambas partículas y las caracteriza explícitamente como chilenismos que son marcadores discursivos. De acuerdo con esta fuente, por ser "se usa para indicar que lo que se dice es un ejemplo que ilustra aquello de lo que se está hablando" (s. v. ser), mientras onda "se usa para indicar que lo que se dirá a continuación es una explicación o una reformulación de lo dicho inmediatamente antes" (s. v. onda). Cabe destacar que el DA y el DUECH también recogen como ser con la misma definición de por ser (s. v. ser). Asimismo, el funcionamiento de ambas partículas en tanto reformuladores explicativos es descrito en Rojas et al. (2012), aunque en otros materiales (Corpus ESECH).

En este contexto, uno de los rasgos que define el modo en que operan los marcadores es su carácter polifuncional, es decir, su capacidad para desempeñar diferentes funciones discursivas (Fuentes 2009) y, en consecuencia, actualizar valores distintos en contextos específicos. Esta polivalencia funcional manifiesta la potencialidad pragmática que poseen 
tales partículas, en pos de guiar la comprensión de los enunciados en los que se insertan y contribuir a la consecución de propósitos comunicativos particulares.

De este modo, a partir de nuestro análisis, hemos determinado que por ser y onda -en tanto marcadores del discurso- cumplen varias funciones. En el corpus PRESEEA-SA hemos verificado las siguientes: 1) explicación, 2) concreción, 3) enunciación y 4) citación. En las secciones que continúan, explicaremos e ilustraremos cada una de ellas.

4.1.1. Reformulador explicativo: los hablantes, mediante el empleo de los marcadores analizados, amplían o re-elaboran un enunciado o concepto anterior, mediante otro enunciado que les permite aclarar mejor una idea. En este caso, por ser y onda son equivalentes a o sea y es decir, como se ilustra en los ejemplos 10 a16:

Por ser

10) E.: y / ¿las características de las personas / de la clase alta? / o mejor dicho / ¿cuáles son las características de los cuicos? I.: eeh / la misma / la misma entrada que tienen / por ser tienen más / debe ser más educación / más desplante / es otro tipo de educación que tiene / son más notorios / son como más cancheros / eso (SCHI_H21_015);

11) E.: ¡la gente! sí ha cambiado mucho // tía y / en su opinión ¿qué es ser un buen amigo? I.: un buen amigo es estar en las buenas y en las malas $<$ silencio/ $>$ $<$ silencio/> por ser si yo / yo te encuentro que estáis mal en el sentido de de / con tu mamá o con tu pololo o con un amiga // no yo te digo "mira yo veo // yo que veo de afuera // veo tu amistad // pero esta es mi opinión / no sé / tú la / si quieres la tomas o la dejas" // o "tu opinión sí / y yo encuentro que no te conviene esa amiga // o este cabro < silencio/> este pololo" // o "tu mamá tiene la razón" (SCHI_M21_021);

12) I.: lo encuentro más / más lógico por ser no sé po / el hombre es un poco como más más fuerte que la mujer / la mujer como dicen es el sexo débil / claro que ahora ha demostrado que no tiene tanta debilidad (SCHI_M32_072);

\section{Onda}

13) I.: sí fumo verde / pero cuando no hay verde igual fumo prensada no tengo / lo tengo que decir / pero hoy día pero ahora como es abril cogollos mil la he pasado súper bien porque me han traído hartos cogollitos y buenos buenos buenos / unos cogollos buenos buenos buenos buenos así onda dos con dos aspiradas y quedáis volada al tiro así no tenís para que seguir fumando (SCHI_M11_007); 
14) I.: sí / me gustaría / tener así como un restaurant ¿cacháis? / o una línea de colectivo onda así / onda que genere plata así / siempre vaya manejándose plata ¿cacháis? // algo // sí algo grande / así un restaurant grande así con comidas lujosas / ¿cacháis? / de otros países y esa onda así (SCHI_H12_041);

15) me busco actividades acá para la noche generalmente y durante el día // descansar mucho / y porque puta que lo valoro / y hacer lo / leseras onda como que // aprovecho el tiempo para hacerlas / para como meterme en los proyectos que no me puedo meter en el año porque no tengo tiempo (SCHI_H13_074);

16) hay como de todo en $\tilde{N}$ / me gusta que / me encanta esa diversidad / me encanta el hecho que hay barrios que son como súper top así como onda edificios ultra modernos todo súper lindo (SCHI_M13_080).

4.1.2. Operador de concreción: los sujetos al emplear por ser y onda introducen un ejemplo, un caso o situación concreta que ejemplifica lo dicho en un enunciado anterior. En esta función las partículas analizadas son conmutables por marcadores como por ejemplo, pongamos por caso o en concreto, tal como se desprende de los ejemplos 17 a 22:

\section{Por ser}

17) I.: pero este de // normal / sí porque yo cuando me ehh // yo presiento cuando mis hermanos están / en algo / o por ser cuando está la N enferma / la C / algo le pasa o la $\mathrm{C} / /$ y después eeh / digo "uuy ¿qué le pasará a la C?" // y al ratito después / mi hermana me llama $<$ silencio/> (SCHI_M21_021);

18) E.: si usted tuviera la oportunidad de cambiarse de comuna / ¿a qué comuna se cambiaría o encuentra que está bien en ésta? I.: no / yo encuentro que estoy bien porque ahora con tanta cosa que está pasando por ser para LC para LB que porque para allá la gente que vive es la que tiene plata / entonces yo en realidad no me cambiaría de barrio me quedaría aquí en mi barrio nomás hasta que Dios me dé vida y salud y me muera / (SCHI_M31_034);

19) I.: a respetarse / claro / por eso ahora por ser el muchacho / se le ocurre que de repente / "ah me voy con los amigos" / va y la deja botada / por qué / porque / se va con los amigos y entonces por eso digo yo que no que no / no sé cómo lo harán po / el pololeo / si es po si es pololeo o o andamos juntos / porque antes no po porque por ser / en el caso mío / yo salía con mi con mi polola / todo el tiempo al lado de ella (SCHI_H32_066);

20) I.: eso claro y de mujeres / a ver / estaba la señora CS // em de también de los antiguos por ser estaba la señora esta locutora eh la de los tangos / ¿cómo se llamaba ésta? eeh AC (SCHI_H32_066); 


\section{Onda}

21) I.: de la $\mathrm{V}$ fue su arpillera eso fue lo y y más sus no sé sus décimas yo creo que también así onda yo creo que hay una que que me gusta mucho que dice eh "mira como se visten cabos y sargentos para teñir de sangre los pavimentos" (SCHI_M11_007);

22) las mejores vacaciones / eeh yo diría que fueron hace como dos años // dos tres años / casi tres años // eeh que me fui con mis dos mejores amigos a LS / eeh nos fuimos a quedar allá una semana / en la casa de las primas de del $\mathrm{U} / \mathrm{mi}$ amigo // y // puta fue / fue rico alejarme tanto de S // estar / onda en LS que es / considerablemente lejos // eeh // en el verano (SCHI_H13_074).

4.1.3. Operador enunciativo con valor continuativo o aproximativo: los hablantes, mediante el empleo de los marcadores analizados, continúan su enunciación, ya sea como una fórmula de relleno que les permite planificar mejor su discurso o indicando que el término empleado no es exacto, sino que cercano a lo que se quiere decir. En este caso, por ser y onda son equivalentes a por decirlo de algún modo o digamos, como se ilustra en 23 a 25:

\section{Por ser}

23) I.: claro porque a ver / compromiso no sería porque entonces ya no sería amistad po / porque resulta que cuando uno es amigo es amigo / pero es que ahora está tan difícil la cosa que uno a veces / y como yo / tal como en mi situación / como yo / donde vivo no no tengo eem / eh por ser amistad con los vecinos / entonces digo yo / de amigos no / ahora ya mayor prácticamente no me preocupo de eso / (SCHI_H32_066);

\section{Onda}

24) I.: fue la experiencia del embarazo fue súper difícil porque quedé embarazada a los diecisiete y y fue yo lo primero que pensé así onda que se había terminado mi vida / porque yo quería seguir haciendo cosas y y un hijo igual era como el miedo po el miedo de enfrentar a ser adulta como al tiro (SCHI_M11_007);

25) I.: en mis años / por ejemplo cuando yo pololeaba / era / nos juntábamos un lote / fumábamos / pero cigarros / éramos onda / éramos sanos po / no éramos lo / locos de esos que fumaban marihuana / no / nos gustaba escuchar música / los C / eeh / puro rock clásico / pero la juventud de antes era mucho mejor / ahora está muy / está muy acelerada la juventud / no me gusta la juventud de ahora (SCHI_M21_019). 
4.1.4. Introductor de discurso directo: los sujetos al emplear por ser y onda introducen una cita en estilo directo. En esta función los marcadores analizados son conmutables por un verbum dicendi como "decir", tal como se desprende de los casos 26 a 29 :

Por ser

26) E.: ¿algún programa en específico? I.: $<$ silencio/ $>\mathrm{mmm} /$ no tanto / aa de repente escucho en la radio $\mathrm{P} /$ las noticias que da $\mathrm{C}$ en la mañana / que llaman por teléfono // dicen las noticias del día / "opinen esto / y llamen" porque opinan de / por ser "¿por qué sube la gasolina? / y ¿qué qué piensa piensa usted? ¿por qué la suben?" / o o / o “¿por qué mataron al G?” o algunas cosas así // entonces dan súper sus opiniones aah ya // "¿cómo era el G antes / eeh / era bueno tenía buena llegada aceptamiento a la gente o no?" // cosas así (SCHI_M21_021);

27) y// y y bueno y se entiende que uno también tiene que saber corresponderle en ese sen / en ese sentido / una persona que no / que no ande pidiendo favores por ser eeh / "oye yo te hago este este este favor a ti pero tú me tienes que hacer / uno a mí" (SCHI_H32_066);

\section{Onda}

28) que tenga buena voluntad con uno // que tenga muy buena voluntad para cualquier cosa / no que sea como ahí flojo y que esté echado y que / uno le diga onda / “ime puede acompañar?” o / no / alguien que sea atento y que / que te ayude y que te sirva / que te sirva / obvio (SCHI_M12_043);

29) los nicks que la gente / pone / que a veces es como // me / onda "no no sé hoy me quiero morir o desearía ay no sé volar lejos" y no sé qué y es como / ya onda "bueno ¿a quién le estáis hablando?" / como que la gente tiene necesidad de de de expresarse (SCHI_M13_080).

Pese a las ventajas de la aplicación del método de la conmutación en el análisis de los marcadores precedentes, a fin de determinar las funciones que comparten por ser y onda, encontramos algunos casos en que la asignación de una función unívoca es compleja, ya que se produce un traslapo de funciones con matices diferenciadores poco claros. Por ejemplo, en 30 y 31 :

30) I.: sí po sí sí / en el metro igual / bueno la última vez que viajé en el metro estaba embarazada / y fue horrible la huevada porque venía lleno ¿cacháis? y me subí a una huevada así que con cueva onda un guardia me ayudó me tuvo que tirar / ayudar a subir y y subí y era tanta la aglomeración de gente huevón que me empecé a desmayar y me tuve que bajar onda a la siguiente estación (SCHI_M11_010); 
31) y yo puta el ahuevonado como andaba en el carro solo cacháis andaba con el / con el discman en esa época / el discman onda afuera así // onda / en el asiento del lado no estaba sentado en el suelo (SCHI_H13_074).

\subsection{ANÁLISIS SOCIOLINGÜÍSTICO DE LOS MARCADORES POR SER Y ONDA EN EL CORPUS PRESEEA-SA}

La Tabla 2 muestra la frecuencia de empleo de las funciones descritas en la sección precedente. Como puede apreciarse, las funciones principales de ambos marcadores son la explicación y la concreción; en cambio, la enunciación y la citación son poco importantes, desde el punto de vista cuantitativo. En términos comparativos, por lo que refiere a sus frecuencias de uso, hemos comprobado que por ser y onda manifiestan comportamientos de sentido inverso. De este modo, aunque más de la mitad de los casos de por ser se pueden atribuir a la función de concreción, la frecuencia de su valor explicativo no está a gran distancia. Por el contrario, el empleo de onda es -en su mayoría- explicativo; por consiguiente, el uso del resto de las funciones resulta ser muy marginal.

Tabla 2. Frecuencia y porcentaje de las funciones de los marcadores por ser y onda identificadas en la muestra

\begin{tabular}{|l|c|c|c|c|}
\cline { 2 - 5 } \multicolumn{1}{c|}{} & \multicolumn{2}{c|}{ Por ser } & \multicolumn{2}{c|}{ Onda } \\
\cline { 2 - 5 } \multicolumn{1}{c|}{} & Frecuencia & $\%$ & Frecuencia & $\%$ \\
\hline Explicación & 12 & 36 & 50 & 71,4 \\
\hline Concreción & 17 & 52 & 8 & 11,4 \\
\hline Enunciación & 2 & 6 & 7 & 10,1 \\
\hline Citación & 2 & 6 & 5 & 7,1 \\
\hline Total & 33 & 100 & 70 & 100 \\
\hline
\end{tabular}

La Tabla 3, por su parte, muestra las frecuencias de uso de los marcadores por ser y onda, de acuerdo con los factores socio-demográficos de los sujetos de la muestra del corpus PRESEEA-SA, esto es, sexo, edad y nivel de instrucción. También, en esta dimensión, ambos marcadores manifiestan comportamientos inversos. Porcentualmente, aunque el empleo de por ser es más frecuente en hombres que en mujeres, el sexo no es determinante en el caso del uso de onda. Igualmente, mientras por ser es más usado por hablantes de mayor edad, onda es preferido por los más jóvenes, casi de modo exclusivo; incluso, no verificamos ejemplos en el grupo de mayor 
edad. Por último, respecto del nivel de instrucción de los sujetos, el empleo de por ser se concentra en el grupo de instrucción medio y no registramos casos en el alto. Por su lado, el uso del marcador onda es más frecuente en el grupo alto, mientras los porcentajes en el medio y en el bajo son cercanos.

Tabla 3. Frecuencia y porcentaje del empleo de los marcadores por ser y onda, según los factores socio-demográficos de los sujetos

\begin{tabular}{|l|c|c|c|c|}
\cline { 2 - 5 } \multicolumn{1}{c|}{} & \multicolumn{2}{c|}{ Por ser } & \multicolumn{2}{c|}{ Onda } \\
\cline { 2 - 5 } \multicolumn{1}{c|}{} & Frecuencia & $\%$ & Frecuencia & $\%$ \\
\hline Hombres & 22 & 66,7 & 39 & 55,7 \\
\hline Mujeres & 11 & 33,3 & 31 & 44,3 \\
\hline $20-34$ & 0 & 0 & 67 & 95,7 \\
\hline $35-54$ & 10 & 30,3 & 3 & 4,3 \\
\hline 55 y más & 23 & 69,7 & 0 & 0 \\
\hline Bajo & 10 & 30,3 & 16 & 22,9 \\
\hline Medio & 23 & 69,7 & 12 & 17,1 \\
\hline Alto & 0 & 0 & 42 & 60 \\
\hline
\end{tabular}

En cuanto a la aplicación de las pruebas de significación estadística, específicamente, la prueba ANOVA, comprobamos que el único contraste de medias significativo fue la edad para el marcador onda en su función como reformulador explicativo. Como se ilustra en el Gráfico 1, los resultados de la aplicación de la prueba de varianza evidencian significación estadística para el empleo de onda explicativo $(\mathrm{F}=7,219, \mathrm{p}=0,01)$, cuyo uso es más frecuente en el grupo etario más joven de los informantes, de acuerdo con el contraste de las medias siguientes: 20-34 (1,333), 35-54 (0,056), 55 y más $(-1,4)$. En este sentido, podemos concluir, con certeza, que el marcador onda explicativo es característico de los sujetos más jóvenes de la comunidad en estudio. 
Gráfico 1. Medias marginales del empleo de onda explicativo y edad de los sujetos de la muestra

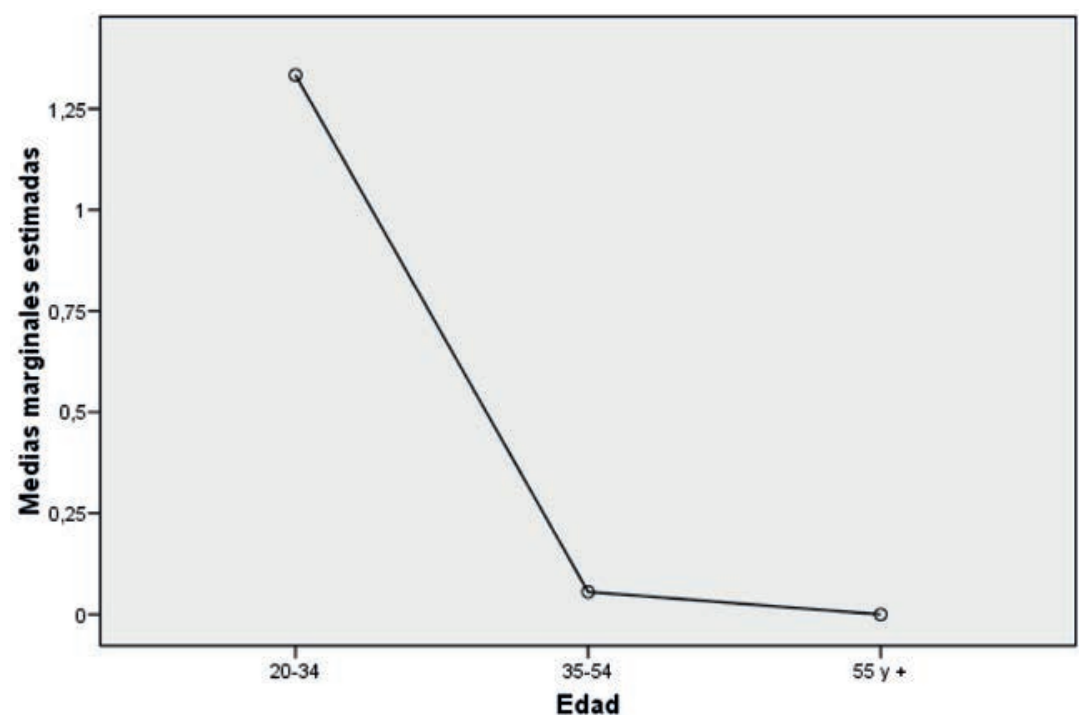

\section{CONCLUSIONES}

En esta investigación hemos analizado el comportamiento pragmático y la estratificación sociolingüística del empleo de los marcadores del discurso por ser y onda, en las 108 entrevistas que conforman el corpus del PRESEEA-SA. Las conclusiones más relevantes de nuestra indagación pueden sintetizarse del siguiente modo:

1. Las locuciones por ser y onda se emplean, en el español de Chile, como marcadores del discurso. Además, en cuanto marcadores, su empleo exhibe la actualización de cuatro funciones discursivas, a saber, 1) explicación, 2) concreción, 3) enunciación y 4) citación. De este modo, se comprueba la primera hipótesis que sustenta nuestra investigación, y que dice relación con el carácter polifuncional que adquieren estas partículas.

2. El análisis cuantitativo general nos permitió concluir que ambas partículas tienen dos funciones principales, a saber, la explicación 
y la concreción. Asimismo, por ser y onda se comportan de manera inversa. Por ser adopta una función prioritaria de concreción, mientras que onda se emplea mayormente con su función explicativa.

3. En relación con la incidencia los factores sociales, al menos en términos porcentuales, también se observó un comportamiento inverso en el empleo de los marcadores por ser y onda. Por una parte, en por ser es más determinante el factor sexo, el que resulta más marginal en el análisis de onda. El contraste etario, por otro lado, muestra que por ser es utilizado por los hablantes de mayor edad y onda por los sujetos más jóvenes. Finalmente, por ser es más utilizado por los hablantes con instrucción media y onda, aunque manifiesta un uso transversal en la muestra analizada, es preferido por los informantes del grupo alto.

4. En cuanto a la aplicación de las pruebas de significación estadística, comprobamos que el único contraste de medias significativo fue la edad, para el marcador onda en su función como reformulador explicativo. En este sentido, se comprueba la segunda hipótesis de nuestro trabajo, según la cual, la incidencia de los factores sociodemográficos en el empleo de los marcadores analizados es desigual.

5. Finalmente, destacamos la relevancia de llevar a cabo trabajos contrastivos con muestras de otras comunidades de habla española, que permitan corroborar si los marcadores por ser y onda son usos diferenciales del español de Chile.

\section{REFERENCIAS BIBLIOGRÁFICAS}

Aschenberg, Heidi y Óscar Loureda Lamas (eds.). 2011. Marcadores del discurso: de la descripción a la definición. Madrid/Frankfurt: Iberoamericana/Vervuert.

Carbonero, Pedro y Juana Santana. 2010. Marcadores del discurso, variación dialectal y variación social. En Óscar Loureda y Esperanza Acín (coords.), pp. 497-521.

CEPEDA, Gladys. 1999. La variación pragmático- discursiva, entonacional y sociolingüística de los conectores conjuntivos en el habla de Valdivia, Chile. En María José Serrano (ed.). Estudios de variación sintáctica. Madrid/Frankfurt: Iberoamericana/Vervuert, pp. 103-120.

Cortés, Luis. 1998. Marcadores del discurso y análisis cuantitativo. En María Martín Zorraquino y Estrella Montolío Durán (coords.), pp. 143-160.

CORTÉs, Luis y MARÍA CAMACHO. 2005. Unidades de segmentación y marcadores el discurso. Madrid: Arco/Libros.

DA=Asociación de Academias de la Lengua Española. 2010. Diccionario de americanismos. Lima: Editorial Santillana. 
DPdE = Briz, Antonio, Salvador Pons y José Portolés (coords.). 2008. Diccionario de partículas discursivas del español. En línea: www.dpde.es.

DUECH= Academia Chilena de la Lengua. 2010. Diccionario de uso del español de Chile. Santiago: Editorial MN.

Fuentes, Catalina. 2009. Diccionario de conectores y operadores del español. Madrid: Arco/Libros.

Hernández Campoy, Juan Manuel y Manuel Almeida. 2005. Metodología de la investigación sociolingüística. Málaga: Editorial Comares.

Labov, William. 1983 (1972). Modelos sociolingüisticos. Madrid: Cátedra.

López Morales, Humberto. 1994. Métodos de investigación lingüística. Salamanca: Ediciones Colegio de España.

LOUREDA, ÓSCAR Y ESPERANZA Acín (coords.). 2010. Los estudios sobre marcadores del discurso, hoy. Madrid: Arco/Libros.

Martín Zorraquino, María. 1998. Los marcadores del discurso desde el punto de vista gramatical. En María Martín Zorraquino y Estrella Montolío (coords.), pp. 19-53.

Martín Zorraquino, María y Estrella Montolío (coords.). 1998. Los marcadores del discurso. Teoría y análisis. Madrid: Arco/Libros.

Martín Zorraquino, María y José Portolés 1999. Los marcadores del discurso. En Ignacio Bosque y Violeta Demonte (eds.). Gramática descriptiva de la lengua española (3 volúmenes). Madrid: Espasa, pp. 4051-4207.

Mondaca, Lissette, Andrea Méndez y Marcela Rivadeneira. 2015. "No es muletilla, es marcador, ¿cachái?". Análisis de la función pragmática del marcador discursivo conversacional cachái en el español de Chile. Literatura y Lingüistica 32: 233-258.

Moreno Fernández, Francisco. 1990. Metodología sociolingüística. Madrid: Editorial Gredos.

Poblete, María Teresa. 1998. Los marcadores discursivo-conversacionales de más alta frecuencia en el español de Valdivia (Chile). Estudios Filológicos 33: 93-103.

1999. La cohesión de los marcadores discursivos en distintos tipos de discurso. Estudios Filológicos 34: 165-180.

Pons, Hernán y José Luis SAmaniego. 1998. Marcadores pragmáticos de apoyo discursivo en el habla culta de Santiago de Chile. Onomázein 3: 11-25.

PoRTolés, José 2001. Marcadores del discurso. Barcelona: Ariel.

Rabanales, Ambrosio y Lidia Contreras. 1995. Las muletillas en el habla culta de Santiago de Chile. Scripta Philologica in honorem Juan M. Lope Blanch, Vol. 2. México: UNAM, pp. 673-744.

Rojas, Cristian, Alejandra Rubio, Abelardo San Martín y Silvana Guerrero. 2012. Análisis pragmático y sociolingüístico del empleo de los marcadores discursivos de reformulación en el habla de Santiago de Chile. Lenguas Modernas 40: 103-123.

RoJAS, Darío. 2008. Funciones actuales y evolución semántica de la locución de repente en el español de Chile. Boletín de Filología XLIII: 207-237.

2012. Huevón como marcador del discurso en el español de chile: huellas de un proceso de gramaticalización. Revista de Humanidades 25: 145-164.

SAn Martín, ABelardo. 2004-2005. Igual como marcador discursivo en el habla de Santiago de Chile: función pragmático-discursiva y estratificación social de su empleo. Boletín de Filología XL: 201-232.

2011. Los marcadores interrogativos de control de contacto en el corpus PRESEEA de Santiago de Chile. Boletín de Filología XLVI (2): 135-166.

2013. Los reformuladores de distanciamiento en el corpus PRESEEA de Santiago de Chile. Boletín de Filología XLVIII (1): 171-199. 
San Martín, Abelardo y Silvana Guerrero. 2015. Estudio Sociolingüístico del Español de Chile (ESECH): recogida y estratificación del corpus de Santiago. Boletín de Filología L (1): 221-247.

Soto, JAIME y YASNA RoldÁn. 2002. Marcadores discursivos en el habla urbana y rural de la provincia de Nuble. Onomázein 7: 87-94.

Valencia, Alba. 2014a. Introducción. Cuadernos de la ALFAL 5: 4-12.

2014b. Marcadores del discurso en Santiago de Chile. Cuadernos de la ALFAL 5: $246-276$ 